\title{
Paired-associate learning in a simple adaptive environment'
}

\author{
MORTON P. FRIEDMAN AND MICHAL C. CLARK ${ }^{2}$ \\ UNIVERSITY OF CALIFORNIA, LOS ANGELES
}

An analysis of paired-associates learning by individual subjects in a "steady state" training situation is presented. The main results concern response correctness and response latency during acquisition and $24 \mathrm{hr}$. recall as a function of controlled practice.

This research dealt with the effects of controlled practice of individual paired-associate items on response correctness and latency during acquisition and recall. A continuous learning task was employed which was designed to (1) allow the examination of the learning process in single Ss over extended periods and (2) to study the learning process in a statistically steady state.

In our "item control" procedure, each item was removed from the practice set and replaced by a new, unlearned item when it reached a certain performance criterion. The number of items which was being practiced was always the same, but there were always items in different stages of learning which provided a "steady state" background against which learning on individual items occurred.

This experimental situation is of interest for several reasons. First, it is more phenotypical of many every day learning situations than is the usual list learning procedure. Second, the "steady state" learning situation would appear to be more appropriate for testing most current models of paired-associate learning than the usual list learning technique in which the learning of items occurs in a systematically changing context. Third, the experimental situation is an example of an adaptive learning environment (Groen \& Atkinson, 1966) which is convenient for studying optimization of the learning process within an individual.

\section{Method}

Both the stimuli and responses were high frequency nouns. Each word appeared in only one S-R pair. Over 3000 S-R pairs were used. The stimuli were presented on $3 \times 5$ in. white index cards. The anticipation method was employed with the correct response always given orally by $E$ after $S$ responded or after 10 sec. if $S$ did not respond. The items were presented in practice sets of $16 \mathrm{~S}-\mathrm{R}$ pairs. After each presentation of the practice set, the order of the items was changed haphazardly. The interval between successive presentations of the set was $25 \mathrm{sec}$. Ss were required to read numbers aloud rapidly between presentations to prevent rehearsal.

As learned items met predetermined performance criteria, they were removed from the practice set and replaced with new items. The practice set was thus kept constant in size. The criterion for item replacement was one, two, or three successive correct responses. The criterion for each item was predetermined randomly, and there were approximately equal numbers of the three types of items. An experimental session consisted of 12 presentations of the practice set. Both immediate and 24-hr. recall tests were given. At the end of each session, Ss were tested for recall on half of the items presented during that session. At the beginning of the next session Ss were tested on the untested remainder of the preceding day's items. No feedback was given on the recall tests. Response latencies during training and testing were recorded using an electronic voice key. Ss responded to the presentation of a stimulus term with one of three responses: They said "new," indicating that they did not recognize the stimulus term and thought that it was part of a new item; "old," indicating that they recognized the stimulus term as belonging to an old item (presented earlier), but they did not recall the correct response; or else they gave the response they recalled as being associated with the stimulus word. The Ss were four male undergraduates who were paid at an hourly rate. Each served for 16 successive daily sessions.

\section{Results and Discussion}

The main findings about response correctness in acquisition will be considered first. To check for changes in rates of learning over sessions, tests were made comparing the total errors per item scores for the first and second half of the sessions for each S. There were no statistically reliable differences $(p<.05)$ and no systematic changes in learning rates over sessions. Accordingly, the results for each $\mathrm{S}$ were pooled across sessions for all analyses.

The data were scored in terms of recognition accuracy of "old" and "new" stimuli before the first correct response on each item. (Response errors are pooled with "old" responses to "old" stimuli in these recognition analyses.) All Ss were quite accurate at discriminating "old" stimuli from "new" stimuli. No $S$ made fewer than $97 \%$ correct recognition responses, and there were no recognition errors at all after the second presentation of a stimulus. For all Ss, the first correct recall response in acquisition occurred very quickly. The mean trial of the first correct recall was $2.4,2.6,2.3$, and 2.2 for the four Ss. For all Ss, once the first correct recall occurred to an item, there were remarkably few errors; the highest error rate after the first correct recall for any $S$ was less than $3 \%$.

Now we consider the response latencies which were 
obtained during acquisition. The important results are shown in Fig. 1. Mean correct response latencies are shown for recognition and recall trials for each of the four Ss. The first point on the abscissa (N) for each $S$ is for the first presentation of a stimulus. Note that this latency is very short for all Ss; thus, not only can Ss correctly identify a new stimulus, but they do it fairly rapidly. The next two points refer to the latencies of the correct recognition responses for old stimuli for the trial before the last error $(-1)$ and for the trial of the last error (0). Compared with the recognition of new stimuli, these latencies are long. Note that the longest latency occurs on the trial of last error. The final three points $(1,2,3)$ are for the latencies on the successive correct recall trials. These latencies are al ways shorter than the error latencies and show a steady decline with increasing number of correct responses. This pattern of latency results is similar to that obtained by Kintsch (1965) and others for paired-associate learning with known responses.

A possible explanation for the observed pattern of latencies is the following: In responding to an item, $S$ first makes a recognition response; if he judges the item to be new, he responds quickly and does not try to retrieve the response. If he recognizes the stimulus as old, the latency is increased because he spends some time trying to retrieve the association. The older the item, the more time he will spend in retrieving the response. There is thus an increase in latency from the next to the last error to the last error. When retrievable, an association is apparently available fairly quickly, and practice makes it available even more quickly. In fact, note that the latency of correct recall on the third successive practice trial is close to the latency of recognition of a new item for all Ss.

It was mentioned earlier that there were no systematic changes in acquisition rate over sessions. However, the 24-hr. recall results show proactive inhibition. For all Ss, the 24-hr. recall probabilities for the first

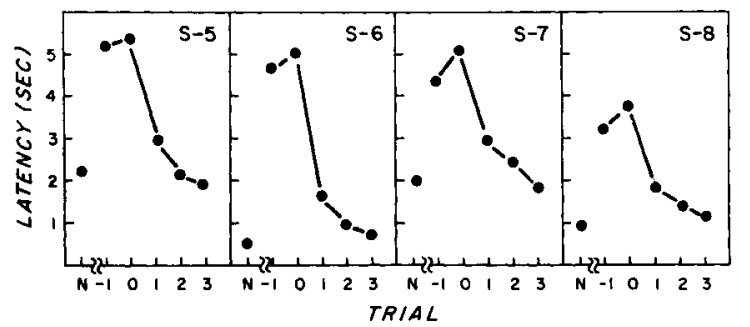

Fig. 1. Response Latency during acquisition. See Text for explanation.

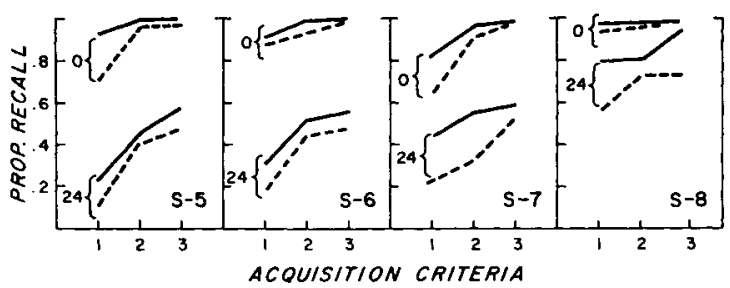

Fig. 2. Proportion of correct recalls on the immediate (0) and 24 hr. tests as a function of acquisition practice level. See text for further explanation.

session a re quite high, but they drop quickly and stabilize after a few sessions. Little or no proactive inhibition was noted in the immediate recall, but this could be due to a "ceiling effect."

Some interesting interrelations between acquisition latency and recall correctness are shown in Fig. 2 for each $\mathrm{S}$ for the immediate and $24-\mathrm{hr}$. recall tests. Recalls are split according to whether the latency on the last practice trial in acquisition was above (broken line) or below (solid line) the median latency for items at each practice level. It can be seen that items whose response latencies were shorter during acquisition are recalled better than items whose latencies were longer. A further analysis of the latencies or the recall tests indicated that items which were responded to faster in acquisition also tended to be given faster during recall.

It is obvious that these data can be fit very comfortably into a Hullian "response strength with a threshold" conception of learning. That recognition of the stimulus occurs even though the response is not made is consistent with the idea that an association has strength below a response threshold. The systematic changes in the error latencies may be interpreted as a "tip of the tongue" effect near the threshold. Also, the decrease in correct response latencies as a function of practice and the effects of practice on recall may both be taken as evidence for the further growth of response strength after it exceeds the recall threshold. There are, of course, other theoretical interpretations we cannot consider here.

\section{References}

Groen, G. J., \& Atkinson, R. C. Models for optimizing the learning process. Psychol. Bull., 1966, 66, 309-320.

Kintsch, W. Habituation of the GSR component of the orienting reflex during paired-associate learning. J. math. Psychol., 1965, $2,330-341$.

\section{Notes}

1. Supported by funds from the U.C.L.A. Research Committee and by N.I.M.H. Grant MH-07809.

2. Now at Stanford University. 